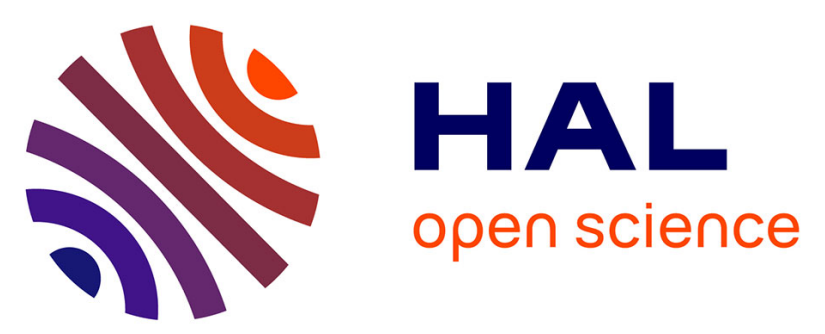

\title{
Theoretical prediction of the onset of thermoacoustic instability from the experimental transfer matrix of a thermoacoustic core
}

Matthieu Guédra, Guillaume Penelet, Pierrick Lotton, Jean-Pierre Dalmont

\section{- To cite this version:}

Matthieu Guédra, Guillaume Penelet, Pierrick Lotton, Jean-Pierre Dalmont. Theoretical prediction of the onset of thermoacoustic instability from the experimental transfer matrix of a thermoacoustic core. Journal of the Acoustical Society of America, 2011, 130 (1), pp.145-152. 10.1121/1.3592227. hal-02057429

HAL Id: hal-02057429

https://hal-univ-lemans.archives-ouvertes.fr/hal-02057429

Submitted on 5 Mar 2019

HAL is a multi-disciplinary open access archive for the deposit and dissemination of scientific research documents, whether they are published or not. The documents may come from teaching and research institutions in France or abroad, or from public or private research centers.
L'archive ouverte pluridisciplinaire HAL, est destinée au dépôt et à la diffusion de documents scientifiques de niveau recherche, publiés ou non, émanant des établissements d'enseignement et de recherche français ou étrangers, des laboratoires publics ou privés. 


\title{
Theoretical prediction of the onset of thermoacoustic instability from the experimental transfer matrix of a thermoacoustic core
}

\author{
Matthieu Guedra, ${ }^{\text {a) }}$ Guillaume Penelet, Pierrick Lotton, and Jean-Pierre Dalmont \\ Laboratoire d'Acoustique de l'Université du Maine, UMR CNRS 6613, Avenue Olivier Messiaen, \\ 72085 Le Mans Cedex 9, France
}

(Received 13 December 2010; revised 6 April 2011; accepted 26 April 2011)

\begin{abstract}
The aim of this paper is to propose a method to predict the onset conditions of the thermoacoustic instability for various thermoacoustic engines. As an accurate modeling of the heat exchangers and the stack submitted to a temperature gradient is a difficult task, an experimental approach for the characterization of the amplifying properties of the thermoacoustic core is proposed. An experimental apparatus is presented which allows to measure the transfer matrix of a thermoacoustic core under various heating conditions by means of a four-microphone method. An analytical model for the prediction of the onset conditions from this measured transfer matrix is developed. The experimental data are introduced in the model and theoretical predictions of the onset conditions are compared with those actually observed in standing-wave and traveling-wave engines. The results show good agreement between predictions from the model and experiments.
\end{abstract}

(C) 2011 Acoustical Society of America. [DOI: 10.1121/1.3592227]

PACS number(s): 43.35.Ud [PBB]

Pages: $145-152$

\section{INTRODUCTION}

The thermoacoustic amplification process results from the thermodynamic interaction between an oscillating fluid and a porous material submitted to a strong temperature gradient. This interaction is responsible for acoustic work generation and hydrodynamic heat transfer. Thermoacoustic engines employ acoustic resonators to take advantage of this interaction in order to produce high amplitude sound waves. ${ }^{1}$ For such engines, there exists a critical temperature gradient along the porous material above which the onset of thermoacoustic instability occurs, leading to the amplification of a self-sustained acoustic wave into the resonator.

One of the key elements of a thermoacoustic engine is the porous material in which the thermoacoustic process occurs. This can be any open-cell porous medium with substantial surface area, high porosity, low thermal conductivity and minimum flow resistivity. In the field of thermoacoustics, this element is referred to as "stack" or "regenerator." When employed as a stack in a standing-wave thermoacoustic engine, it has to ensure an imperfect heat transfer between the oscillating fluid and the adjacent solid walls (the nominal pore diameter must be on the order of magnitude of a few thermal acoustic boundary layer thicknesses). When employed as a "regenerator" in a traveling-wave thermoacoustic engine ${ }^{2}$ (also called thermoacoustic Stirling engine ${ }^{3}$ ), the pores must be sufficiently thin to ensure a quasi-isothermal contact between the solid walls and the oscillating fluid. The early thermoacoustic engines employed periodic structures such as parallel stainless steel plates ${ }^{4}$ or honeycombed materials, ${ }^{5}$ but recent advances in the development of thermoacoustic engines involve using more unusual structures like pin-array stacks, ${ }^{6}$ Reticulated Vitreous Carbon foam (RVC) ${ }^{7}$ or stacked stainless steel screens. ${ }^{3}$ Con-

\footnotetext{
a) Author to whom correspondence should be addressed. Electronic mail: matthieu.guedra.etu@univ-lemans.fr
}

ventional stacks arrangement like parallel plates or uniform stacks of arbitrary cross-sectional geometry can be described in the frame of the "capillary tube based" theory, ${ }^{8-10}$ where the viscous and thermal coupling between the stack and the oscillating fluid is described by means of thermoacoustic functions $f_{\kappa}$ and $f_{\nu}$, which depend on the specific channel geometry under consideration. Substantial experimental and theoretical works have been led for the description of more complicated structures. In a series of papers, ${ }^{11-14}$ Wilen and Petculescu measured the thermoacoustic functions for various pore geometries and stack arrangements (including RVC and aluminum foam) from the experimental complex density and complex compressibility, with or without a temperature gradient. Muehleisen et al. ${ }^{15}$ performed a four-microphone method to measure the complex characteristic impedance and wave number for RVC. Roh et $a l .{ }^{16}$ developed a mathematical model of sound propagation in a porous material with an imposed temperature gradient which was successfully compared to the experimental data of Wilen and Petculescu, ${ }^{13,14}$ after scaling using dynamic shape factors and tortuosity.

While the efficiency of thermoacoustic engines depends critically on the properties of the stack (or regenerator), numerous other parameters control the operation of the engine. In particular, when trying to predict the onset of thermoacoustic instability, the knowledge of the characteristics of the stack is not sufficient. For instance, the heat exchangers are responsible for a flow resistance which must be considered. The distribution of the temperature field along the thermoacoustic core [see Fig. 1(b)] also needs to be known accurately, as it has been demonstrated that the shape of the temperature field (notably in the thermal buffer tube) strongly impacts the thermoacoustic amplification process. ${ }^{17}$ Due to this, an accurate theoretical description of the onset conditions of a thermoacoustic engine should involve the description of the heat exchangers and a complete model including the description of the (three-dimensional) 
(a)
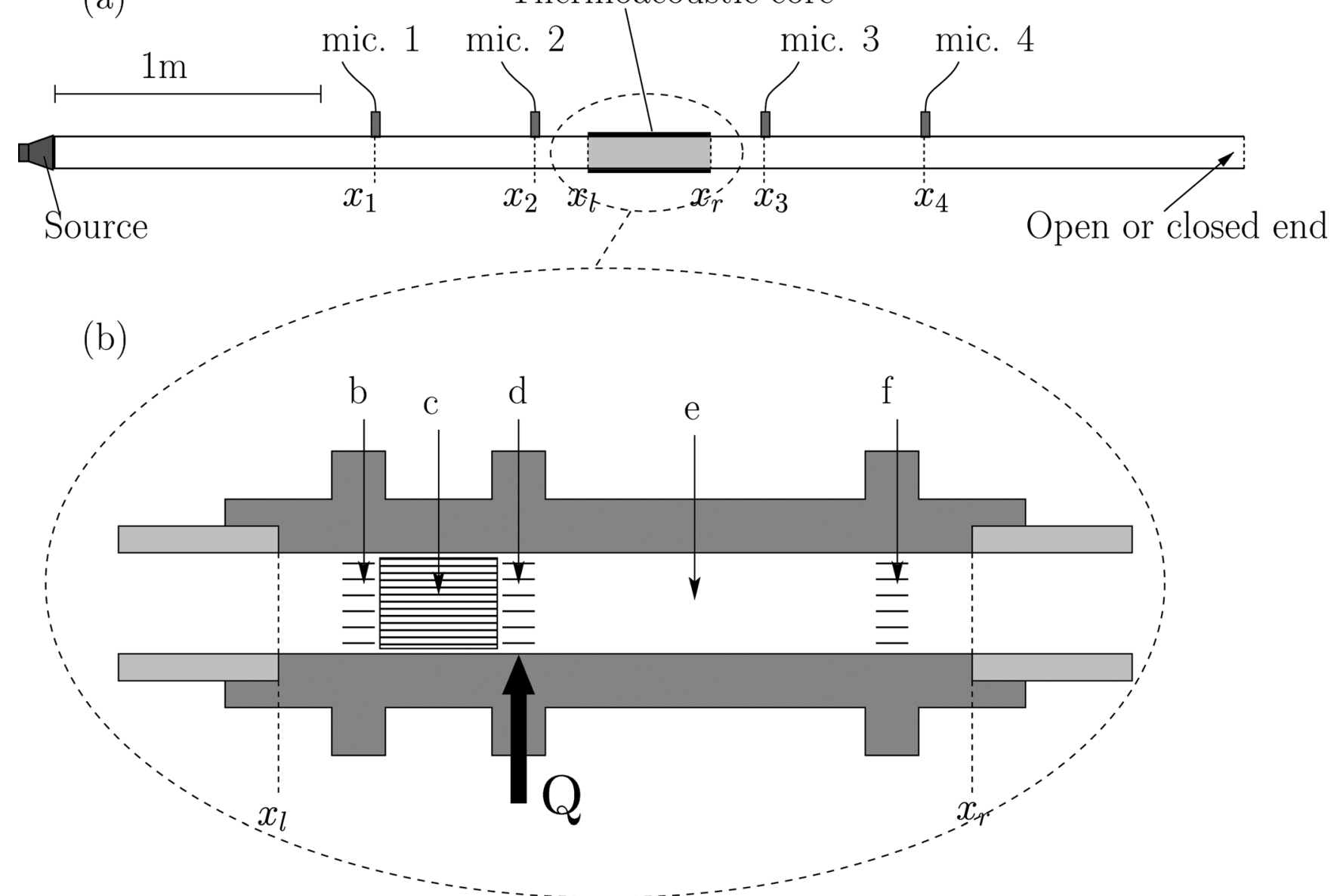

FIG. 1. (a) Scale drawing of the experimental apparatus used to measure the transfer matrix of the thermoacoustic core. (b) Drawing of the thermoacoustic core under study which is constituted of a cold exchanger (a), the stack (b), a hot exchanger (c) which supplies a heat power $Q$ to the system, the thermal buffer tube (d) and a second cold exchanger (e).

temperature field inside the thermoacoustic core. Note that an accurate description of the engine's operation after the onset of thermoacoustic instability should also include the description of numerous nonlinear effects saturating the wave amplitude growth. ${ }^{18-21}$ That is the reason why the design and the optimization of the existing prototypes are generally done partly with the help of available softwares based on the linear (or weakly nonlinear) thermoacoustic theory, ${ }^{22}$ and partly in a semiempirical way.

The purpose of this paper is to present an alternative method for the determination of the onset conditions of the thermoacoustic instability for various thermoacoustic engines. This method consists in considering the whole thermoacoustic core as an unknown two-port whose transfer matrix is measured by means of a "two-load" method. ${ }^{24,25}$ The advantage of this method is to avoid the description of the geometric complication of the stack and the heat exchangers as well as the temperature distribution. In Sec. II, the experimental setup is presented, which permits to measure the transfer matrix of the thermoacoustic core on a frequency range from 50 to $200 \mathrm{~Hz}$ and for several heating power $Q$ supplied to the hot heat exchanger. Once experimentally determined, this transfer matrix is used to predict the onset conditions for various engines in which the thermoacoustic core is inserted. In order to derive these onset conditions, a theoretical modeling is presented in Sec. III, which can be applied to various kinds of thermoacoustic engines, including standing- or traveling-wave engines (in the presence of an acoustic load or not). In Sec. IV, the experimental data are included in the model and the resulting predictions of the onset conditions (in terms of heating power supply $Q$ and acoustic frequency $f$ ) of thermoacoustic instability are compared with those actually observed when the thermoacoustic core is mounted into standing-wave and traveling-wave thermoacoustic engines.

\section{MEASUREMENT OF THE TRANSFER MATRIX OF THE THERMOACOUSTIC CORE}

A schematic drawing of the experimental apparatus used for the determination of the transfer matrix of a thermoacoustic core is shown in Fig. 1(a). The thermoacoustic core under study [see Fig. 1(b)] is a 46-cm-long stainless steel waveguide of inner radius $16.7 \mathrm{~mm}$. The stack (b) is a 7.2-cm-long ceramic sample with square pores of semiwidth $0.6 \mathrm{~mm}$. A temperature gradient is applied along the stack by means of two heat exchangers: a hot exchanger (c) which supplies a heat power $Q$ to the system and a cold exchanger (a) which ensures that the left side of the stack is maintained at room temperature. A second cold exchanger 
(e) is used to control the temperature distribution along the thermal buffer tube (d). The cold heat exchangers are made up of two copper pipes passing through a honeycombed aluminum disk, with flowing water inside the pipes at room temperature. The hot heat exchanger is made up of a piece of ceramic sample (the same as the one used for the stack) in which a Nichrome resistance wire is coiled. The thickness of the sample is $1 \mathrm{~cm}$ and the heat resistance wire is connected to an electrical DC power supply controlling the heat power $Q$ dissipated by Joule effect into the wire.

In order to characterize the propagation of acoustic waves through the thermoacoustic core, two straight PVC ducts of 2-m-length are connected to its ends [see Fig. 1(a)]. The duct on the left is connected to an electrodynamic loudspeaker, while the duct on the right is either open to free space or closed with a rigid wall. Two pairs of microphones (model Bruel and Kjaer 4136) are flush mounted along each duct at both sides of the thermoacoustic core. Assuming that harmonic plane waves are propagating in the waveguide at angular frequency $\omega$, the acoustic pressure $p(x, t)$ and acoustic volume velocity $u(x, t)$ are written as

$$
\zeta(x, t)=\Re\left[\tilde{\zeta}(x) e^{-j \omega t}\right]
$$

where $\zeta$ may be either $p$ or $u, \sim$ denotes the complex amplitude and $\Re()$ denotes the real part of a complex number. With this assumption, the complete apparatus can be used to measure the complex amplitudes of acoustic pressures $\tilde{p}\left(x_{l, r}\right)$ and acoustic volume velocities $\tilde{u}\left(x_{l, r}\right)$ at left side and right side of the two-port, respectively, and thus to deduce the transfer matrix of the thermoacoustic core $\mathbf{T}_{T C}$ using the following relation

$$
\begin{aligned}
\left(\begin{array}{c}
\tilde{p}\left(x_{r}\right) \\
\tilde{u}\left(x_{r}\right)
\end{array}\right) & =\left(\begin{array}{cc}
\mathcal{T}_{p p} & \mathcal{T}_{p u} \\
\mathcal{T}_{u p} & \mathcal{T}_{u u}
\end{array}\right) \times\left(\begin{array}{c}
\tilde{p}\left(x_{l}\right) \\
\tilde{u}\left(x_{l}\right)
\end{array}\right) \\
& =\mathbf{T}_{T C} \times\left(\begin{array}{c}
\tilde{p}\left(x_{l}\right) \\
\tilde{u}\left(x_{l}\right)
\end{array}\right) .
\end{aligned}
$$

The measurement method used here to obtain $\mathbf{T}_{T C}$ is a classical "two-load," four-microphone method. ${ }^{24,25}$ Note that some multiple pressure sensor methods has already been used in the field of thermoacoustics: ${ }^{26,27}$ for instance, Petculescu and Wilen ${ }^{27}$ employed a four sensor method to measure the input and output acoustic powers through a regenerator with an imposed temperature gradient. However, to our knowledge the multiple microphone method has not been used to measure the transfer matrix of a thermoacoustic core. The basic principles of this method consists in calculating $\tilde{p}\left(x_{l, r}\right)$ and $\tilde{u}\left(x_{l, r}\right)$ from the measurements of acoustic pressures at positions $x_{i}[i=1,2,3,4$, see Fig. 1(a)]. A set of two measurements is however required to obtain the four coefficients $\mathcal{T}_{p p}, \mathcal{T}_{p u}, \mathcal{T}_{u p}, \mathcal{T}_{u u}$ of the transfer matrix from the measured acoustic pressures $\tilde{p}\left(x_{i}\right)$, and this is realized here by using two different acoustic loads, the first with the open duct, and the second with the duct closed by a rigid wall. This four-microphone method, which has been exten- sively used in the field of guided-wave acoustics, is further described in the Appendix.

The measurements of the transfer matrix $\mathbf{T}_{T C}$ are operated here for 26 different values of heat power $Q$ supplied to the hot heat exchanger, from $0 \mathrm{~W}$ to $83 \mathrm{~W}$, and in the frequency range from 50 to $200 \mathrm{~Hz}$. The viscous and thermal penetration depths, $\delta_{\nu}$ and $\delta_{\kappa}$, respectively, are given for this frequency range, at room temperature $(300 \mathrm{~K})$ :

$$
\begin{aligned}
& 0.31 \mathrm{~mm}>\delta_{\nu}>0.16 \mathrm{~mm} \\
& 0.38 \mathrm{~mm}>\delta_{\kappa}>0.19 \mathrm{~mm}
\end{aligned}
$$

For a given heat power $Q$, the electrodynamic loudspeaker is excited with a swept sine signal. The frequency increment of the swept sine signal is fixed to $1 \mathrm{~Hz}$, and the microphone signals are sampled at $10 \mathrm{kHz}$. The magnitude and phase of the acoustic pressures measured by the microphones are estimated using a least-square method.

It is worth noting that the method used here for the determination of $\mathbf{T}_{T C}$ is a strongly indirect method and it is thus important to bring many precautions in order to prevent from large errors. For instance, it is important to ensure airtightness at the locations where the ducts, the thermoacoustic core and the microphones are connected each other (this is realized using vacuum grease). The microphone separation $\Delta x$ is chosen according to the frequency range of analysis and fixed to $\Delta x=60 \mathrm{~cm}$ in order to satisfy the criterium given by $\mathrm{Abom}^{24}$

$$
0.1 \pi<k_{0} \Delta x<0.8 \pi
$$

where $k_{0}=\omega / c_{0}$ is the acoustic wave number and $c_{0}$ stands for adiabatic sound velocity. Attention must also be paid to the relative calibration of the microphones in order to prevent from large errors due to inherent differences between the sensors in terms of sensitivity and phase shift (small differences between the sensors may lead to large errors in the estimate of $\mathbf{T}_{T C}$ ). ${ }^{24}$ This relative calibration (and the subsequent corrections of the measurements) is realized by exposing them to the same sound field in a small cavity, and by measuring their cross-spectra in the frequency range of interest. $^{29}$

As an example, Fig. 2 presents the four coefficients $\mathcal{T}_{p p}$, $\mathcal{T}_{p u}, \mathcal{T}_{u p}$ and $\mathcal{T}_{u и}$ (amplitude and phase) of the transfer matrix from 50 to $200 \mathrm{~Hz}$ and for three different values of heat power $Q$. For $Q=0 \mathrm{~W}$, the mean value of the amplitude of the determinant $\mathcal{T}_{p p} \mathcal{T}_{\text {uи }}-\mathcal{T}_{p u} \mathcal{T}_{\text {up }}$ over the frequency range is $0.998\left(\approx 1\right.$, with a standard deviation $\left.\sigma \approx 9.10^{-3}\right)$ and the mean value of the argument is $-3 \cdot 7 \cdot 10^{-3} \mathrm{rad}(\approx 0 \mathrm{rad}$, with a standard deviation $\sigma \approx 5 \cdot 2 \cdot 10^{-3} \mathrm{rad}$ ), which confirms that the transfer matrix of the two-port is reciprocal when no temperature gradient is applied along the stack. The influence of $Q$ on the transfer properties of the thermoacoustic core is clearly visible: the four coefficients significantly change in both amplitude and phase when $Q$ is increased. It is also notable that the experimental curves are smooth on the whole frequency range without any visible measurement artifact. In order to prevent from an overloading of the graphs, errorbars have not been plotted in Fig. 2, but the uncertainties on the 


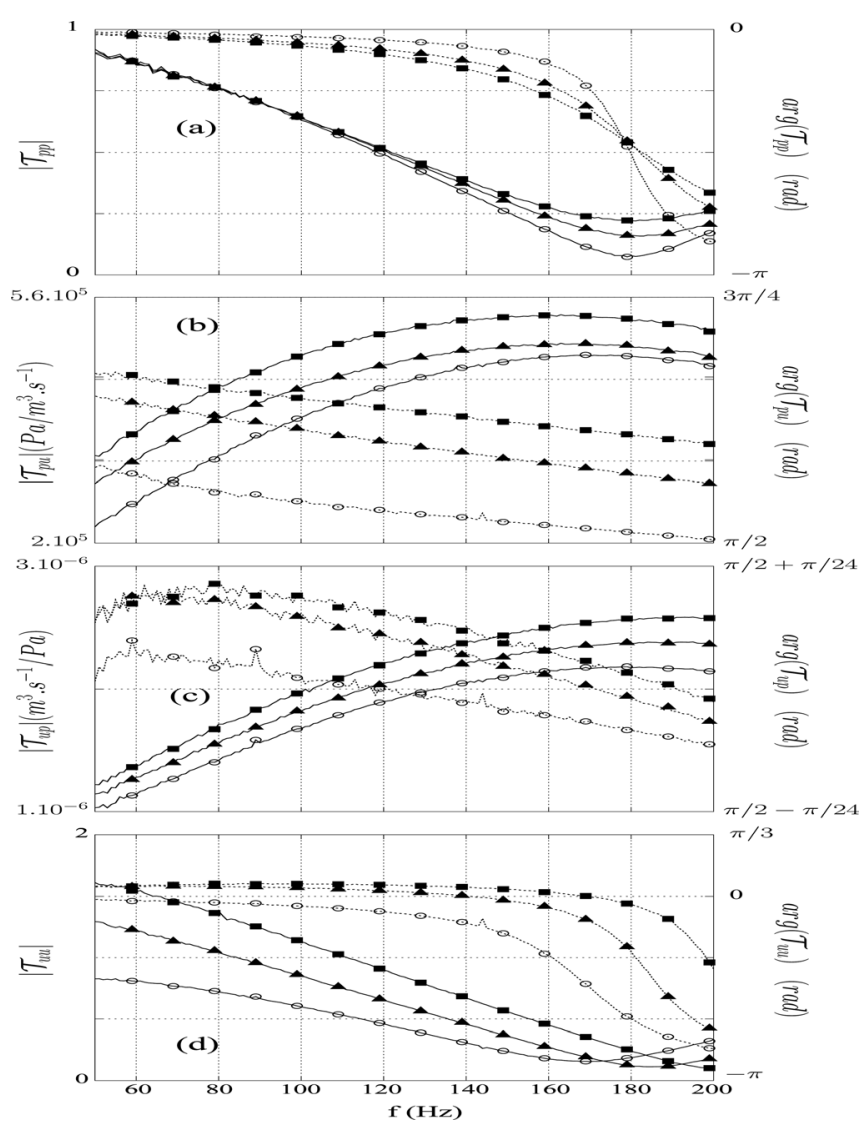

FIG. 2. Amplitude (-) and phase (...) of the coefficients $\mathcal{T}_{p p}$ (a), $\mathcal{T}_{p u}$ (b), $\mathcal{T}_{u p}$ (c) and $\mathcal{T}_{\text {ии }}$ (d) as functions of the frequency $f$ for several increments of heat power $Q \cdot Q_{\min }=0 \mathrm{~W}(\circ), Q=36 \mathrm{~W}$ (solid triangle) and $Q_{\max }=83 \mathrm{~W}$ (solid square).

room temperature $(\Delta T \approx 0.5 \mathrm{~K})$, the static pressure $(\Delta P \approx 1$ mbar), the inner radius of the apparatus $(\Delta R \approx 0.1 \mathrm{~mm})$, the distances between microphones $(\approx 0.5 \mathrm{~mm})$, and the uncertainties in the least-square estimates of acoustic pressures $\tilde{p}\left(x_{i}\right)$ impact the calculation of $\mathbf{T}_{T C}$. The resulting relative errors on the coefficients $\mathcal{T}\left(\mathcal{T}=\mathcal{T}_{p p}, \mathcal{T}_{p u}, \mathcal{T}_{u p}\right.$ and $\left.\mathcal{T}_{u u}\right)$ have been quantified, leading to $\Delta|\mathcal{T}| /|\mathcal{T}| \approx 2 \%$ and $\Delta \arg (\mathcal{T}) \approx 2.10^{-2} \mathrm{rad}$ in the whole frequency range.

It is worth noting that the experiments were made here at moderate acoustic pressure levels in order to prevent from the influence of nonlinear effects (nonlinear propagation, thermoacoustic heat transport and acoustic streaming). More precisely, the electrical voltage supplied to the loudspeaker was sufficiently low in order that the peak amplitudes of acoustic pressure in the frequency range of interest were comprised between $0.1 \mathrm{~Pa}$ and $8 \mathrm{~Pa}$ (the highest pressure amplitude measured at one of the resonances of the complete apparatus). In order to be sure that nonlinear effects do not influence the measurements, we made additional measurements at lower acoustic levels and we checked that the resulting transfer matrix components shown in Fig. 2 stayed unchanged. It is also worth noting that because the two load method employed here is based on the assumption of linear acoustic propagation, the obtained transfer matrices cannot be used for the prediction of the phenomena which occur after the onset of thermoacoustic instability.

\section{CALCULATION OF THE ONSET CONDITIONS FROM THE TRANSFER MATRIX OF THE THERMOACOUSTIC CORE}

\section{A. Standing-wave engine}

A standing-wave thermoacoustic engine is generally made up of a straight acoustic resonator inside which the thermoacoustic core is mounted, as shown in Fig. 3(a). The propagation of acoustic waves through the thermoacoustic core is determined from the experimental results presented in Sec. II which lead to the transfer matrix $\mathbf{T}_{T C}$ of the thermoacoustic core [Eq. (2)]. $\mathbf{T}_{T C}$ captures the specific geometry and thermophysical properties of the different components (stack and heat exchangers) and depends notably on the angular frequency $\omega$ and on the temperature distribution $T(x)$ along the core, which is directly linked to the heat power $Q$ supplied to the hot exchanger. The prediction of the onset of thermoacoustic instability involves finding conditions on $\omega$ and $Q$, i.e., finding the critical heat power $Q_{\text {onset }}$ necessary for the growth of a sound wave, together with the corresponding angular frequency $\omega_{\text {onset }}$ of the selfsustained thermoacoustic oscillations.

While $\mathbf{T}_{T C}$ is determined from experiments, the theoretical modeling of the complete device requires to describe the acoustic propagation through the components surrounding the thermoacoustic core [see Fig. 3(a)]. Writing the propagation of harmonic plane waves in the wave guides of respective lengths $x_{l}$ and $L-x_{r}$ leads to the expressions of the impedances

$$
\begin{aligned}
& Z_{l}=\frac{\tilde{p}\left(x_{l}\right)}{\tilde{u}\left(x_{l}\right)}=\frac{Z_{0}+j Z_{c} \tan \left(k x_{l}\right)}{1+j Z_{0} Z_{c}^{-1} \tan \left(k x_{l}\right)}, \\
& Z_{r}=\frac{\tilde{p}\left(x_{r}\right)}{\tilde{u}\left(x_{r}\right)}=\frac{Z_{L}-j Z_{c} \tan \left[k\left(L-x_{r}\right)\right]}{1-j Z_{L} Z_{c}^{-1} \tan \left[k\left(L-x_{r}\right)\right]},
\end{aligned}
$$

loading both sides of the thermoacoustic core, where $k=k_{0} \epsilon_{\text {loss }}$ and $Z_{c}$ are the complex wave number and the characteristic impedance of the wave guide defined in Appendix by Eqs. (A2) and (A9), respectively, and where the boundary conditions at $x=0$ and $x=L$ are accounted for by means of impedance conditions

$$
\begin{aligned}
& \tilde{p}(0)=Z_{0} \tilde{u}(0), \\
& \tilde{p}(L)=Z_{L} \tilde{u}(L) .
\end{aligned}
$$

The acoustic impedances $Z_{0}$ and $Z_{L}$ can be, for instance, the radiation impedance of an open pipe, ${ }^{30}$ the infinite impedance of a rigid wall, or the acoustic impedance of an electrodynamic alternator, depending on the configuration of the standing-wave engine. Finally, combining Eqs. (5) and (6) with Eq. (2) and solving the associated system of two equations leads to the equation

$$
\tilde{F}(\omega, Q)=0
$$

where the function $\tilde{F}$ is defined as

$$
\tilde{F}=Z_{l} \mathcal{T}_{p p}+\mathcal{T}_{p u}-Z_{l} Z_{r} \mathcal{T}_{u p}-Z_{r} \mathcal{T}_{u u}
$$


(a)

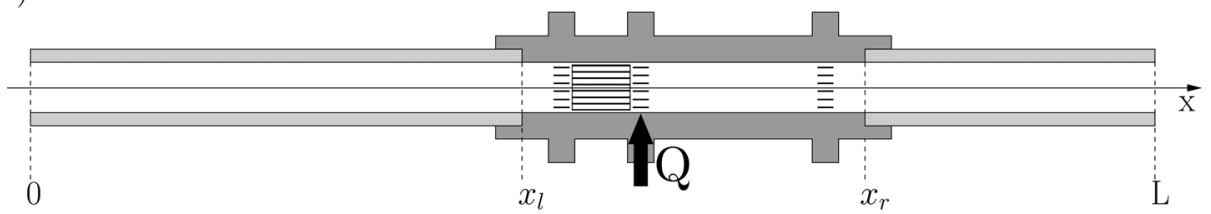

(b)

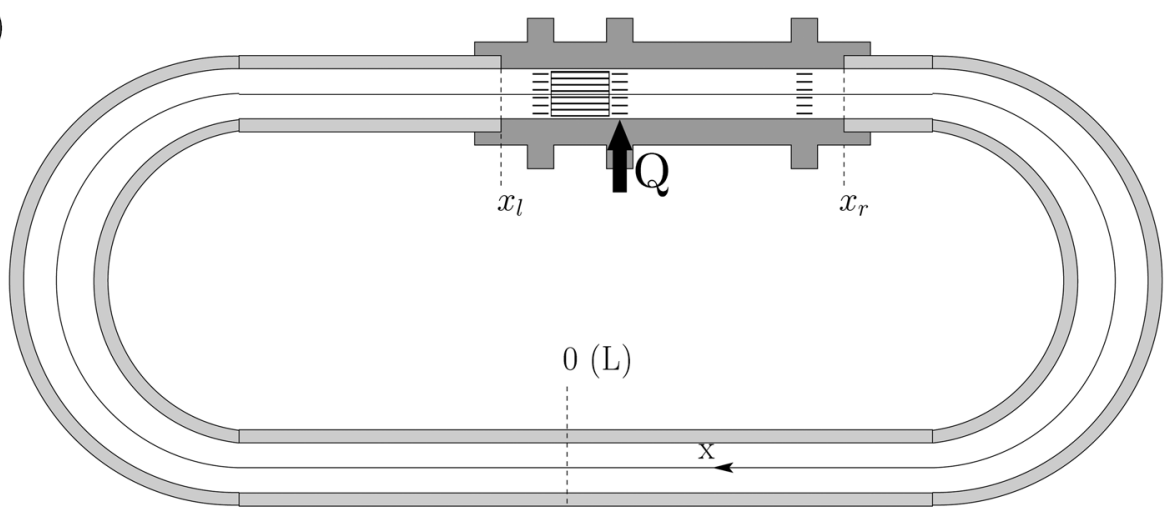

(c)

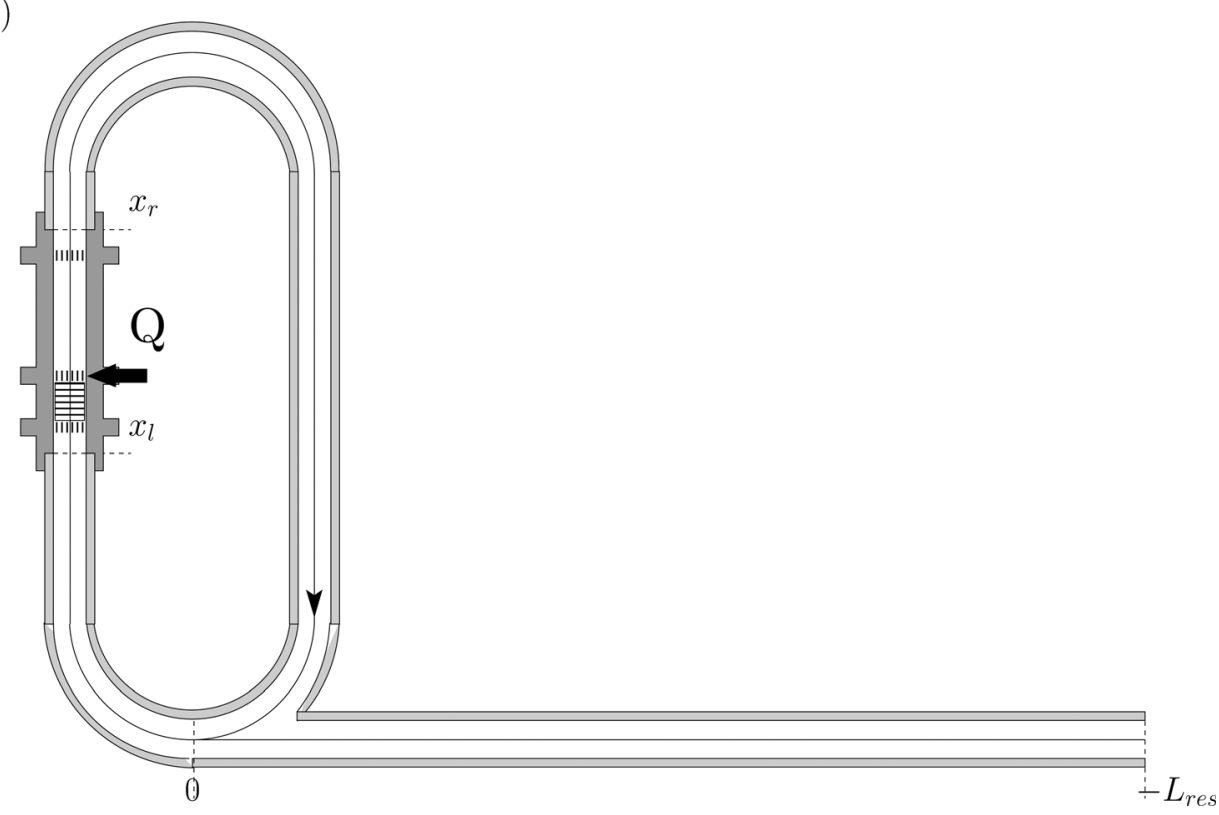

FIG. 3. Drawing of a standing-wave engine (a) and of a closed-loop traveling-wave engine coupled (c) or not (b) with a secondary resonator.

Determining the onset conditions implies finding the appropriate $\omega_{\text {onset }}$ and $Q_{\text {onset }}$ for which Eq. (9) is satisfied. In practice, this is carried out by finding the pair of values $(\omega, Q)$ which minimizes the modulus of the complex function $\tilde{F}^{31}$ As the matrix components are obtained here for discrete values of $\omega$ and $Q$, the onset parameters correspond to experimental values and no interpolation was made on experimental data to obtain the results presented in Sec. IV. However, the frequency and heat power resolutions on measurements have been taken into account in the estimate of uncertainties for $\omega_{\text {onset }}$ and $Q_{\text {onset }}$.

\section{B. Closed-loop traveling-wave engine}

A closed-loop traveling-wave engine is drawn in Fig. 3, depending on whether it is coupled [Fig. 3(c)] or not [Fig. 3 (b)] with a secondary resonator. Note that the configuration (c) is similar to the one investigated by Biwa et al., ${ }^{23}$ who observed a transition from the "standing-wave" mode (onset frequency close to the eigen frequency of the straight tube alone) to the "traveling-wave" mode (onset frequency close to the eigen frequency of the closed-loop waveguide) as $Q$ was increased.

The propagation of a plane wave in the closed loop is supposed to be equivalent to the propagation in a straight duct with the same unwrapped length (the effects due to the loop curvature are assumed to be negligible) and the coupling of the annular resonator with a secondary acoustic load [as, for example, a straight resonator as shown in Fig. 3(c)] can be introduced by an equivalent acoustic impedance in the continuity equations as

$$
\tilde{p}(0)=\tilde{p}(L)=\tilde{p}_{e q},
$$




$$
\tilde{u}(0)=\tilde{u}(L)-\tilde{u}_{e q}=\tilde{u}(L)-Z_{e q}^{-1} \tilde{p}(L),
$$

where $Z_{e q}=\tilde{p}_{e q} / \tilde{u}_{e q}$ is the impedance of the acoustic load. In the case of a straight resonator of length $L_{r e s}$, it can be written as

$$
Z_{e q}=\frac{Z_{\mathrm{lim}}-j Z_{c, r e s} \tan \left(k_{\text {res }} L_{r e s}\right)}{1-j Z_{\mathrm{lim}} Z_{c, \text { res }}^{-1} \tan \left(k_{\text {res }} L_{r e s}\right)}
$$

where $Z_{\lim }$ is the ending impedance at $x=-L_{\text {res }}$ and can be replaced with the radiation impedance of an open pipe ${ }^{30}$ or the infinite impedance of a rigid wall. Note that $Z_{c, \text { res }}$ and $k_{\text {res }}$ are the characteristic impedance and the complex wave number in the straight resonator, respectively, and can be different from $Z_{c}$ and $k$ as the inner radius of the pipe can be different from the inner radius of the loop.

Then, using the propagation of acoustic waves in the loop combined with Eq. (2) and Eqs. (11) and (12) and solving the associated set of equations leads to Eq. (9), with the following expression for the function $\tilde{F}$ :

$$
\begin{aligned}
\tilde{F}= & 1+\mathcal{T}_{p p} \mathcal{T}_{u u}-\mathcal{T}_{p u} \mathcal{T}_{u p}-\cos \left(k L_{w}\right)\left(\mathcal{T}_{p p}+\mathcal{T}_{u u}\right) \\
& -j \sin \left(k L_{w}\right)\left(Z_{c}^{-1} \mathcal{T}_{p u}+Z_{c} \mathcal{T}_{u p}\right)+\Psi
\end{aligned}
$$

where $L_{w}=L-x_{r}+x_{l}$, and where $\Psi$ represents the contribution of the secondary acoustic load. If the device under consideration is a closed-loop engine [Fig. 3(b)], then $\Psi=0$, and if the closed loop is coupled with a secondary resonator, then

$$
\Psi=\frac{Z_{c}}{Z_{e q}} \sum_{i=1}^{2} \sum_{j=1}^{2}\left(\mathbf{M}_{i j} \mathbf{T}_{T C_{i j}}\right)
$$

In Eq. (15), the matrix $\mathbf{M}$ is defined as

$$
\mathbf{M}=\left[\begin{array}{c}
\cos \left(k d_{r}\right) \\
j Z_{c} \sin \left(k d_{r}\right)
\end{array}\right] \times\left[\begin{array}{c}
-j \sin \left(k x_{s}\right) \\
-Z_{c}^{-1} \cos \left(k x_{s}\right)
\end{array}\right]^{t},
$$

where $t$ denotes the transposed vector, and where $d_{r}=$ $L-x_{r}$.

Finally, it is possible to derive analytically the function $\tilde{F}(\omega, Q)$ for various thermoacoustic engines. The onset conditions, $\omega_{\text {onset }}$ and $Q_{\text {onset }}$, can then be determined from the experimental transfer matrix of the thermoacoustic core $\mathbf{T}_{T C}$ obtained in Sec. II by minimizing the modulus of $\tilde{F}$.

\section{PREDICTIONS OF THE ONSET CONDITIONS}

Once experimentally determined, the transfer matrix of the thermoacoustic core $\mathbf{T}_{T C}$ is included in the model in order to predict the onset conditions for two engine configurations: a standing-wave engine [Fig. 3(a)] and a travelingwave engine coupled with a secondary straight resonator [Fig. 3(c)]. Then, in order to evaluate the accuracy of this combined analytical-experimental model, each engine is built and the actual onset conditions $\left(f_{\text {onset }}, Q_{\text {onset }}\right)$ are measured.

\section{A. Standing-wave engine}

The study is first made for a straight "open-closed" standing-wave engine [see Fig. 3(a)]. The thermoacoustic core characterized beforehand is now connected to two straight cylindrical PVC pipes with the same inner radius. The pipe on the left side is closed and its length is kept constant at $L-x_{r}=10 \mathrm{~cm}$. The other pipe is open to free space and its length $x_{l}$ varies from $17 \mathrm{~cm}$ to $1 \mathrm{~m}$. Figure 4 represents the onset frequency $f_{\text {onset }}$ (upper graph) and the onset heat power supply $Q_{\text {onset }}$ (lower graph) of the "open-closed" standing-wave engine as functions of the length $x_{l}$. Open circles (o) correspond to the experimental data while filled circles $(\bullet)$ correspond to the theoretical data. It can be noticed that $f_{\text {onset }}$ decreases when the total length of the engine increases, as the onset frequency of this standingwave engine remains close to the quarter-wavelength resonance frequency of the "open-closed" pipe.

The theoretical results are in good agreement with those actually observed in terms of both frequency $f_{\text {onset }}$ and heat input $Q_{\text {onset }}$. Theoretical values are quite lower than the experimental ones, but the evolution of $f_{\text {onset }}$ and $Q_{\text {onset }}$ is well reproduced by the model. The plotted errorbars on the analytical results come from the frequency and heat power resolution but also, as discussed in Sec. II, from the uncertainties on the room temperature, on the atmospheric pressure, on the inner radius of the apparatus, on the distances between microphones, and on the measured acoustic pressures (amplitude and phase). The resulting errors on the transfer coefficients (and thus on the function $\tilde{F}$ ) are calculated using the classical uncertainty propagation law.

\section{B. Traveling-wave engine}

Predictions from the model are also compared with the onset conditions actually observed for a closed-loop engine coupled with a secondary straight resonator [see Fig. 3(c)]. The total length of the loop is $L=2.4 \mathrm{~m}$ and the distance
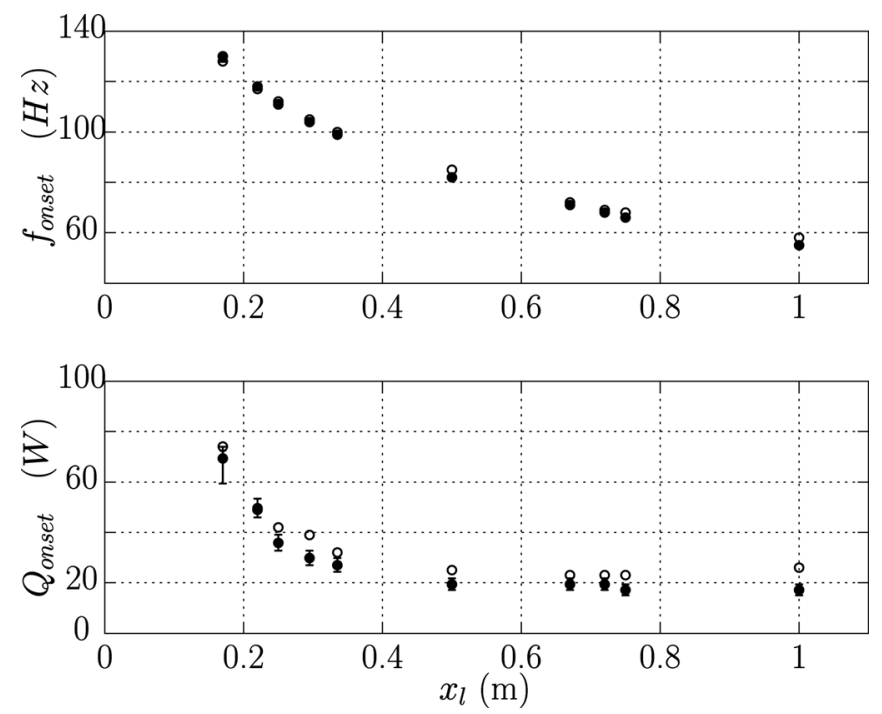

FIG. 4. Onset conditions of an "open-closed" standing-wave engine as functions of the length $x_{l}$ : $f_{\text {onset }}$ (upper graph) and $Q_{\text {onset }}$ (lower graph). The predictions from the semi-analytical model are represented by filled symbols $(\bullet)$ while experimental data are represented by open symbols (०). 

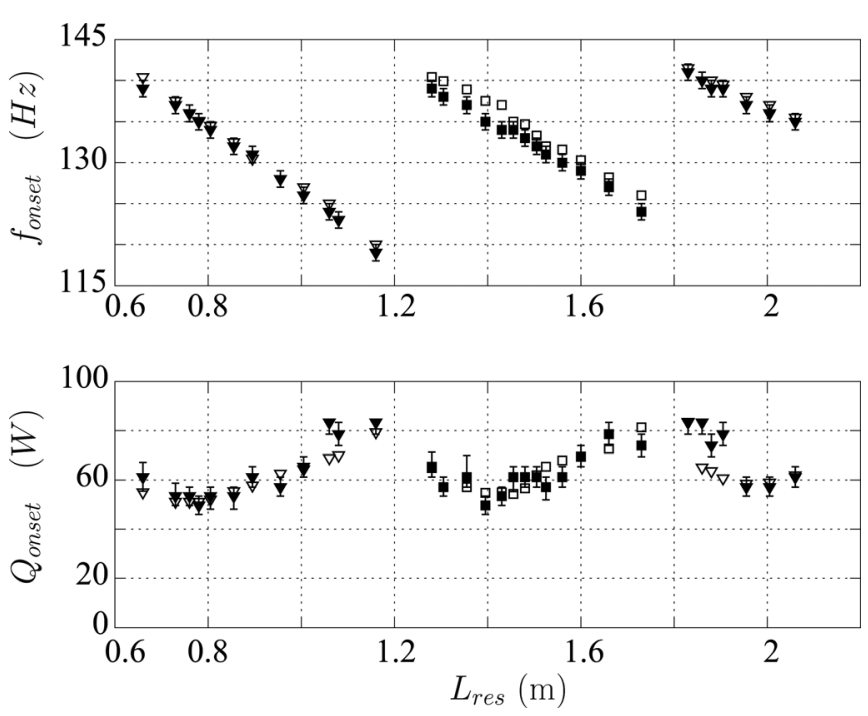

FIG. 5. Onset conditions of traveling-wave engines as functions of the length $L_{\text {res }}: f_{\text {onset }}$ (upper graph) and $Q_{\text {onset }}$ (lower graph). Open triangles represent the results for a loop engine coupled with an open-ending resonator, while open squares represent the results for a loop engine coupled with a closed-ending resonator. The predictions from the semi-analytical model are represented by filled symbols while experimental data are represented by open symbols.

between the cold exchanger and the T-junction is $0.65 \mathrm{~m}$. In Fig. 5, the onset conditions are presented as functions of the length $L_{\text {res }}$ of the straight resonator: triangles represent the results obtained when the resonator is open at its end while squares represent the results for the closed resonator. It is interesting to observe that, depending on the value of $L_{\text {res }}$, the potential amplification of the thermoacoustic instability is governed by the type of impedance at the end of the secondary resonator: the open end is more favorable for $L_{\text {res }} \simeq[0.5,1.2] \cup[1.8,2.1] \mathrm{m}$ while the closed end is more favorable for $L_{\text {res }} \simeq[1.2,1.8] \mathrm{m}$. Note that both cases were investigated for every values of $L_{\text {res }}$. The absence of values for some lengths is due to a too high onset threshold (experimentally as well as analytically), clearly above the maximum heat power $Q_{\max }=83 \mathrm{~W}$ which can be supplied to the hot exchanger.

As for the case of a standing-wave engine, the model shows good results for the traveling-wave engines, in particular, for the onset frequency which is well predicted. There are however larger relative uncertainties on $Q_{\text {onset }}$ coming from a more important propagation of errors in the calculation of $\tilde{F}$.

\section{CONCLUSION}

Finding onset conditions in a thermoacoustic engine requires to be able to characterize many complex elements such as the heat exchangers and the stack submitted to a temperature gradient. The method presented in this paper, which considers the thermoacoustic core as a "black box," is an alternative to the complete analytical description of a thermoacoustic core from its geometrical and thermophysical properties.

The transfer matrix $\mathbf{T}_{T C}$ of the thermoacoustic core is measured by means of a "two-load," four-microphone method, on a frequency range from 50 to $200 \mathrm{~Hz}$ and for several heating power $Q$ supplied to the hot heat exchanger. The onset conditions are then calculated from these experimental results by means of an analytical model describing the acoustic propagation in the components surrounding the thermoacoustic core. An analytical expression of a complex function $\tilde{F}(\omega, Q)$ is derived from the coefficients of the matrix $\mathbf{T}_{T C}$ and the onset conditions $\left(\omega_{\text {onset }}, Q_{\text {onset }}\right)$ are obtained by minimizing the modulus of $\tilde{F}$. The model developed in this work is suitable for the introduction of the experimental transfer matrix of the thermoacoustic core which allows to predict onset conditions without making any assumptions on geometrical properties of the stack or on the shape of the temperature field. Moreover, this model permits to derive onset conditions for standing-wave engines as well as traveling-wave engines coupled with secondary acoustic loads.

The comparison of experimental and theoretical data shows good agreement, which demonstrates that both the measurements of the transfer matrix and the derivation of the analytical model are consistent. Works will be devoted in the future to the experimental characterization of more complicated stacks or regenerators (such as RVC foams or stacked stainless steel screens for instance) and to the application of the present method to the determination of onset conditions in more complicated thermoacoustic engines such as, for instance, a thermoacoustic Stirling engine equipped with an electrodynamic alternator for power production. ${ }^{28}$ The present studies are a first step in future works devoted to the description of thermoacoustic cores, where the measured transfer matrices will be used to validate a model including both linear acoustic propagation and heat transfer in the thermoacoustic core. Some unknown parameters could be determined using an inverse method so that the theoretical transfer matrix fits the experimental one. Then these parameters can be used in a model describing the wave amplitude growth and saturation due to nonlinear processes (with subsequent prediction of the engine's efficiency).

\section{ACKNOWLEDGMENTS}

We would like to thank Joël Gilbert for many useful advices concerning the manuscript.

\section{APPENDIX: TWO-LOAD METHOD}

Considering the apparatus shown in Fig. 1(a) and assuming that the inner radius of the ducts is much smaller than the acoustic wavelength, the acoustic pressure can be separated into two counterpropagating plane waves, so that the complex amplitude of the acoustic pressure is written as

$$
\begin{aligned}
\tilde{p}(x)= & \tilde{p}^{+}(x)+\tilde{p}^{-}(x) \\
= & \tilde{p}^{+}\left(x_{l}\right) e^{j k\left(x-x_{l}\right)}+\tilde{p}^{-}\left(x_{l}\right) e^{-j k\left(x-x_{l}\right)} \\
& \forall x \in\left[0, x_{l}\right],
\end{aligned}
$$

where

$$
k=k_{0} \sqrt{\frac{1+(\gamma-1) f_{\kappa}}{1-f_{\nu}}}=k_{0} \epsilon_{\text {loss }}
$$


is the complex wave number which accounts for viscous and thermal losses in the vicinity of the duct walls and $x_{l}$ corresponds to the left side of the thermoacoustic core. In Eq. (A2), $c_{0}$ and $\rho_{0}$ stand for adiabatic sound velocity and fluid density, $k_{0}=\omega / c_{0}$, and the functions $f_{\nu}$ and $f_{\kappa}$ characterize the viscous and thermal coupling between the oscillating fluid and the walls..$^{8-10}$ In the same way, the pressure on the right side is given by

$$
\tilde{p}(x)=\tilde{p}^{+}\left(x_{r}\right) e^{j k\left(x-x_{r}\right)}+\tilde{p}^{-}\left(x_{r}\right) e^{-j k\left(x-x_{r}\right)} \forall x \in\left[x_{r}, L\right],
$$

where $x_{w}$ corresponds to the right side of the thermoacoustic core. From Eq. (A1) and Eq. (A3), the four counterpropagating components of acoustic pressures on both sides of the thermoacoustic core

$$
\begin{aligned}
& \tilde{p}^{+}\left(x_{l}\right)=\frac{\tilde{p}_{2} e^{j k\left(x_{l}-x_{1}\right)}-\tilde{p}_{1} e^{j k\left(x_{l}-x_{2}\right)}}{2 j \sin \left[k\left(x_{2}-x_{1}\right)\right]}, \\
& \tilde{p}^{-}\left(x_{l}\right)=\frac{\tilde{p}_{1} e^{-j k\left(x_{l}-x_{2}\right)}-\tilde{p}_{2} e^{-j k\left(x_{l}-x_{1}\right)}}{2 j \sin \left[k\left(x_{2}-x_{1}\right)\right]}, \\
& \tilde{p}^{+}\left(x_{r}\right)=\frac{\tilde{p}_{4} e^{-j k\left(x_{3}-x_{r}\right)}-\tilde{p}_{3} e^{-j k\left(x_{4}-x_{r}\right)}}{2 j \sin \left[k\left(x_{4}-x_{3}\right)\right]}, \\
& \tilde{p}^{-}\left(x_{r}\right)=\frac{\tilde{p}_{3} e^{j k\left(x_{4}-x_{r}\right)}-\tilde{p}_{4} e^{j k\left(x_{3}-x_{r}\right)}}{2 j \sin \left[k\left(x_{4}-x_{3}\right)\right]},
\end{aligned}
$$

are expressed as functions of the measured acoustic pressures $\tilde{p}_{1}=\tilde{p}\left(x_{1}\right), \tilde{p}_{2}=\tilde{p}\left(x_{2}\right), \tilde{p}_{3}=\tilde{p}\left(x_{3}\right)$, and $\tilde{p}_{4}=\tilde{p}\left(x_{4}\right)$ at the location of each microphone.

By measuring the pressure signals (amplitude and phase) from each microphone, it is thus possible to estimate $\tilde{p}^{ \pm}\left(x_{l}\right)$ and $\tilde{p}^{ \pm}\left(x_{r}\right)$, and then to deduce the transfer matrix $\mathbf{T}_{T C}$ of the thermoacoustic core [Eq. (2)] by using Eq. (A1) and Euler's equation ${ }^{1}$

$$
\tilde{u}(x)=\frac{S\left(1-f_{\nu}\right)}{j \omega \rho_{0}}\left(\frac{\partial \tilde{p}}{\partial x}\right)=\frac{1}{Z_{c}}\left[\tilde{p}^{+}(x)-\tilde{p}^{-}(x)\right],
$$

where

$$
Z_{c}=\frac{\rho_{0} c_{0}}{S \sqrt{\left(1-f_{\nu}\right)\left[1+(\gamma-1) f_{\kappa}\right]}},
$$

is the characteristic impedance of the waveguide of cross section $S$.

However, it clearly appears that Eq. (2) is equivalent to a system of two equations with four unknown variables (the four matrix coefficients). It is thus necessary to make two measurements with two different configurations of the experimental setup. ${ }^{25}$ The "two-load" method used here consists in making one measurement with a first acoustic load at the right end of the duct [see Fig. 1(a)], and then a second measurement with a different acoustic load.

${ }^{1}$ G.W. Swift, "Thermoacoustics - A unifying perspective for some engine and refrigerators," Acoust. Soc. Am. 113, 2379-2381 (2003).
${ }^{2}$ P. Ceperley, “A pistonless Stirling engine - the traveling wave heat engine," J. Acoust. Soc. Am. 66(5), 1508-1513 (1979).

${ }^{3}$ S. Backhaus, and G.W. Swift, "A thermoacoustic Stirling heat engine," Nature 399, 335-338 (1999).

${ }^{4}$ A.A. Atchley, "Standing wave analysis of a thermoacoustic prime-mover below onset of self-excitation," J. Acoust. Soc. Am. 92(5), 2907-2914 (1992).

${ }^{5}$ G.W. Swift, "Analysis and performance of a large thermoacoustic engine," J. Acoust. Soc. Am. 92(3), 1551-1563 (1992).

${ }^{6}$ G. W. Swift and R. M. Keolian, "Thermoacoustics in pin-array stacks," J. Acoust. Soc. Am. 94(2), 941-943 (1993).

${ }^{7}$ J. A. Adeff, T. J. Hofler, A. A. Atchley, and W. C. Moss, "Measurements with reticulated vitreous carbon stacks in thermoacoustic prime movers and refrigerators," J. Acoust. Soc. Am., 104(1), 32-38 (1998).

${ }^{8}$ N. Rott, "Thermoacoustics," Adv. Appl. Mech. 20, 135-175 (1980).

${ }^{9}$ G. W. Swift, “Thermoacoustic engines," J. Acoust. Soc. Am. 84(4), 11451179 (1988).

${ }^{10}$ W. P. Arnott, H. E. Bass, and R. Raspet, "General formulation of thermoacoustics for stacks having arbitrarily shaped pore cross sections," J. Acoust. Soc. Am. 90(6), 3228-3237 (1991).

${ }^{11}$ L. A. Wilen, "Measurements of thermoacoustic functions for single pores," J. Acoust. Soc. Am. 103(3), 1406-1412 (1998).

${ }^{12}$ G. Petculescu and L. A. Wilen, "Thermoacoustics in a single pore with an applied temperature gradient," J. Acoust. Soc. Am. 106(2), 688-694 (1999).

${ }^{13} \mathrm{~L}$. A. Wilen, "Dynamic measurements of the thermal dissipation function of reticulated vitreous carbon," J. Acoust. Soc. Am. 109(1), 179-184 (2001).

${ }^{14} \mathrm{~A}$. Petculescu, and L. A. Wilen, "Lumped-element technique for the measurement of complex density," J. Acoust. Soc. Am. 110(4), 1950-1957 (2001).

${ }^{15}$ R. T. Muehleisen, C. W. Beamer, I. V. and B. D. Tinianov, "Measurements and empirical model of the acoustic properties of reticulated vitreous carbon," J. Acoust. Soc. Am. 117(2), 536-544 (2005).

${ }^{16}$ H. Roh, R. Raspet, and H.E. Bass. "Parallel capillary-tube-based extension of thermoacoustic theory for random porous media," J. Acoust. Soc. Am. 121(3), 1413-1422 (2007).

${ }^{17}$ G. Penelet, S. Job, V. Gusev, P. Lotton, and M. Bruneau, "Dependence of sound amplification on temperature distribution in annular thermoacoustic engines," Acust. Acta Acust. 91(3), 567-577 (2005).

${ }^{18} \mathrm{H}$. Yuan, S. Karpov, and A. Prosperetti, "A simplified model for linear and nonlinear processes in thermoacoustic prime movers. part 2: nonlinear oscillations," J. Acoust. Soc. Am. 102(6), 3497-3506 (1997).

${ }^{19}$ S. Karpov and A. Prosperetti, "A nonlinear model of thermoacoustic devices," J. Acoust. Soc. Am. 112(4), 1431-1444 (2002).

${ }^{20}$ M. F. Hamilton, Y. A. Ilinskii, and E. A. Zabolotskaya, "Nonlinear twodimensional model for thermoacoustic engines," J. Acoust. Soc. Am. 111(5), 2076-2086 (2002).

${ }^{21}$ G. Penelet, V. Gusev, P. Lotton, and M. Bruneau, "Experimental and theoretical study of processes leading to steady-state sound in annular thermoacoustic engines," Phys. Rev. E 72(1), 016625 (2005).

${ }^{22}$ W. C. Ward and G. W. Swift, "Design environment for low-amplitude thermoacoustic engines," J. Acoust. Soc. Am. 95(6), 3671-3672 (1994).

${ }^{23}$ T. Biwa, Y. Ueda, T. Yazaki, and U. Mizutani. "Thermodynamical mode selection rule observed in thermoacoustic oscillations," Europhys. Lett. 60(3), 363-368 (2002).

${ }^{24} \mathrm{H}$. Boden and M. Abom, "Influence of errors on the two-microphone method for measuring acoustic properties in ducts," J. Acoust. Soc. Am. 79(2), 541-549 (1986).

${ }^{25}$ M. L. Munjal. Acoustics of Ducts and Mufflers With Application to Exhaust and Ventilation System Design (Wiley-interscience, New York, 1987), 352 pages.

${ }^{26}$ A. M. Fusco, W. C. Ward, and G. W. Swift. "Two-sensor power measurements in lossy ducts," J. Acoust. Soc. Am. 91(4), 2229-2235 (1992).

${ }^{27}$ G. Petculescu, and L.A. Wilen. "Traveling-wave amplification in a variable standing wave ratio device," ARLO 3(2), 71-76 (2002).

${ }^{28} \mathrm{~S}$. Backhaus, E. Tward, and M. Petach, "Traveling-wave thermoacoustic electric generator," Appl. Phys. Lett. 85(6), 1085-1087 (2004).

${ }^{29}$ G. Krishnappa, "Cross-spectral method of measuring acoustic intensity by measuring phase and gain mismatch errors by microphone calibration," $J$. Acoust. Soc. Am. 69(1), 307-310 (1981).

${ }^{30}$ J.-P. Dalmont, C. J. Nederveen, and N. Joly, "Radiation impedance of tubes with different flanges: Numerical and experimental investigations," J. Sound Vib. 244(3), 505-534 (2001).

${ }^{31}$ J. K. Hale and H. Kocak. Dynamics and Bifurcations (Springer-Verlag, New York, 1991), Chap. 2, pp. 26-65. 